\title{
Aerodigestive Invasive Malignancy
}

National Cancer Institute

\section{Source}

National Cancer Institute. Aerodigestive Invasive Malignancy. NCI Thesaurus. Code C155820.

A finding indicating the presence of an invasive malignant neoplasm involving the respiratory tract and the upper digestive tract. 